\title{
Infants' early competence for language and symbols
}

\author{
G Dehaene-Lambertz' ${ }^{1}$ A Flo ${ }^{1} \&$ M. Pena² \\ 1: INSERM, UMR992; CEA, NeuroSpin Center; University Paris Saclay, Gif-sur-Yvette, \\ France \\ 2: Pontificia Universidad Católica de Chile, Santiago, Chile
}

\begin{abstract}
Humans have much more sophisticated communication skills than other species. They are not limited to emotional cries, alarm calls and soothing demands, but they interpret the inner and outer world in a symbolic way, resulting in a collective intelligence and an accumulation of knowledge called culture. This culture permeates the child and fosters efficient learning, based on the knowledge accumulated through generations. To develop this collective intelligence, it requires (1) a social brain predisposed to learn from conspecifics, (2) awareness of one's mental state and knowledge, and those of others, (3) a shared common language of though, (4) a communication system for exchanging this information. In this essay, we insist on the value of symbolic representations as a compressed necessary format for representing information to ourselves and exchanging information with others. We propose that human cognition has been boosted beyond the cognition of other primates by the multiplicative advantage of co-development of social cognition, language but also symbolic thinking that can be observed from the first months of life on.
\end{abstract}


Humans are constantly looking for rules and causal relationships to explain what happened and predict what will happen. Collaborative thinking in adults allows a significant improvement in predictions accuracy (Bahrami et al., 2010), but collaborating with others requires, on the one hand, having explicit representations of the problem to resolve and, on the other hand, knowing that it is possible to share these representations unambiguously with another mind. This shared cognition implies a set of symbols that efficiently summarize the concepts we want to represent first to ourselves and second to share with others, but also an implicit assumption that the other can understand these symbols in order to capitalize on each other knowledge. Thus, beyond a theory of mind, this shared cognition requires a pedagogical stance, as proposed by Csibra and Gergely (2009). This pedagogical capacity might have existed since the ancient hominins, if we accept the Oldowan stone tool industry (2.34 million years ago) (d'Errico \& Banks, 2015) as one of the oldest testimony of collective elaborated production. But how does it begin in infants?

\section{A symbolic brain}

In the flow of thoughts, to isolate relevant information that could be shared with others, it is necessary to summarize and discretize sensory information. A first step is to gather different objects sharing common characteristics into a single category, but a more powerful operation would be to further compress this information into a single arbitrary symbolic form. Humans are particularly skilled at creating and using symbolic systems: music notation, traffic signs, equations, even scarification and uniforms are simplified marks that summarize complex information. Language is the first symbolic system acquired by infants and the most productive and versatile. A word can condense the essence of an individual, a category of objects, an action, an abstract concept as freedom. These symbols can be combined in logical operations such as addition, negation, exclusion, quantification or even superimposed to create poetic effects. The symbolic power of language is evident in adult exchanges. Infants may also be sensitive to it very early on, when they listen to speech. 
No one denies that words are arbitrary labels attached to a semantic concept, but the initial relationship between the label and the concept is discussed. It is conventionally assumed that, because a label is produced associated with an object, infants first learn about the co-occurrence of these two events, the labels being only another characteristic of the object, such as the sound it makes when it falls. Gradually, infants understand that the label can be used to refer to the object. Instead, we propose that infants immediately use the label as an internal variable that stands for the object. We also propose that this variable is explicit, at a highlevel node that establishes contact between a global workspace and domain-specific modules. Because of the location of symbols at a higher-level, infants can explicitly and consciously control their use of labels, notably to share and receive information. They can also use them to combine concepts calculated in underlying modules, such as "to the left of the blue wall", generating new unitary representations (HermerVazquez, Spelke, \& Katsnelson, 1999). We support our proposal on comparative brain anatomy, on a reinterpretation of published studies in infants, and on recent studies directly testing the hypothesis of an early symbolic system.

\section{Development of the frontal areas in humans}

Symbolic representations and manipulation are assumed to be supported by frontal areas (Nieder, 2009). Indeed, when adult humans and macaques listen to the same tone sequences varying either on the number of tones or on the structure of the sequence, both species detect the changes but only in humans, a common region, the left inferior frontal gyrus, is activated by both changes. This result was interpreted as evidence of a more abstract "change" code in humans compared to a simple response of discrimination in each specific module in macaques (Wang, Uhrig, Jarraya, \& Dehaene, 2015).

These cross-species computational differences are supposed to be supported by the expansion of the associative areas of the frontal lobe, the inferior parietal and the posterior part of the superior temporal regions (Chaplin, Yu, Soares, Gattass, \& Rosa, 2013) in parallel with the development of large tracts connecting the frontal areas to all other lobes, such as the arcuate fasciculus with the inferior 
parietal and the superior temporal regions. The major difference in connectivity between macaques and humans is the large connectivity of the inferior frontal regions with the associative auditory cortices in humans (Neubert, Mars, Thomas, Sallet, \& Rushworth, 2014).

These particularities are already observed during gestation and the development of the human brain differs significantly from monkeys and even from older humans, in particular its prolonged maturation over many years, which increases the period of plasticity. Unlike chimpanzees, human fetuses retain rapid brain growth after 22 weeks of gestation, which persists even up to 2 years (Sakai et al., 2012; DeSilva \& Lesnik, 2006; Coqueugniot, Hublin, Veillon, Houet, \& Jacob, 2004; Neubauer, Gunz, \& Hublin, 2010). During that period, the prefrontal cortex develops faster than the rest of the brain, again unlike chimpanzees (Sakai et al., 2011). Even compared to ancient homo sapiens, the globular shape of the modern human brain is more pronounced and develops mainly after birth. Endocasts of sapiens and Nehandertal newborns are not very different, while adult shapes differ due to the enlargement of integration cortices (Neubauer, Hublin, \& Gunz, 2018; Gunz, Neubauer, Maureille, \& Hublin, 2010)

However, because of their slow maturation, associative regions have long been thought to be poorly functional at a young age and the role of frontal regions in infant cognition has been underestimated. Yet, several functional MRI studies have shown early activations in these areas. Moreover, as any other brain region, the frontal lobe is never activated as a whole but is parceled into regions that show functional similarity with adult responses. For example, when short-term verbal memory is required, inferior frontal regions are involved (Dehaene-Lambertz, Dehaene, \& Hertz-Pannier, 2002) whereas attention to long-term memory content depends on more dorsal regions (Dehaene-Lambertz et al., 2006). Similarly to adults, the balance between medial prefrontal and orbito-frontal regions is observed in infants when a familiar rewarding stimulus, such as the maternal voice, and a new and unknown stimulus with a value to evaluate, such as the voice of another mother, are presented (Dehaene-Lambertz et al., 2010). 
Frontal areas are not only at the top of a hierarchy of a bottom-up flow of information, they also send feedback information to improve perception and direct learning. The hierarchical organization of brain areas defined by the relative proportion of neurons in supra-granular (contributing to feed-forward pathways) and infra-granular (contributing to feedback pathways) layers is observed since gestation in primates. Feed-forward axons reach the correct target before the end of gestation. By contrast, feed-back connectivity is exuberant and progressively pruned after birth (Price et al., 2006). Evidence of top-down activity has been observed with near infrared spectroscopy in 8-month-old infants, who were exposed to pairs of a tone followed by a smiley. From time to time the smiley was absent. Nevertheless, a response in the visual areas was recorded revealing that infants were expecting the image (Emberson, Richards, \& Aslin, 2015). Other experiments using EEG also show complex expectations in infants at 5 months (Kabdebon \& Dehaene-Lambertz, 2019) and 12 months (Kouider et al., 2015) after they have learned arbitrary soundimages associations.

Frontal neurons through long-distance connectivity participate in a powerful global workspace that offers the possibility of integrating the results of the computations of the many modular brain networks into a common space (Mesulam, 1998; Dehaene \& Naccache, 2001). Information in this central space can be maintained for a long time, amplified and combined with other information but at the cost of slow serial entries. Moreover, these entries can be consciously manipulated, that is they become explicit for oneself and reportable to others.

The signature of this conscious space is an all-or-none response. If a stimulus dimension is linearly manipulated, such as the duration of presentation of a masked face, the sensory cortices follow the same linear response. In infants, the amplitude and duration of the P400 vary linearly with the duration of the face presentation. By contrast, later responses are only recorded when the stimulus is consciously perceived and not when it remains below the perception threshold displaying a characteristic all-or-none response. In adults, this conscious stage is reached in 300 ms while it is around one second in infants (Kouider et al., 2013). 
Therefore, the immaturity of the child's brain which is often apprehended as a contrast between mature low-level regions and immature high-level regions, is more appropriately described as a dynamic competition between parallel circuits whose computational efficiency is controlled by maturation. Differences in the speed of local computations and information transfer can favor one circuit over the other, in particular to enter the global workspace and be amplified and integrated into explicit representations.

In summary, maturation extends over many years in humans, refining connectivity and accelerating local computations and information exchange between distant regions, but the neural architectural design similar to that of adults provides identical or similar computational properties, but at a much slower speed. Notably abstract computations, such as the manipulation of symbols and the conscious access to mental representations, can be accessible even to very young children. Do we have evidence of such abilities?

\section{The power of words in infants}

The first acquired symbolic system is language and many studies point that infants by 5 to 6 months of age, if not earlier, might be equipped with a functional referent-label mapping mechanism. As early as 6 months, they have already noticed a few words and associate them with people ("mon" "dad" the infant's nameTincoff \& Jusczyk, 1999), body parts (Tincoff \& Jusczyk, 2012) and actions ("hug", "eat" Bergelson \& Swingley, 2012). In the lab, they easily learn to map arbitrary sounds to objects: at 2-months, one syllable with one familiar object for example but very quickly they succeed in more complex tasks (Gogate, 2010; Gogate, Prince, \& Matatyaho, 2009). Using ERP, Friedrich and Friederici $(2011,2017)$ reported that 3month-old and 6-month-old infants were able to learn the mapping between 8 words and objects. However, only older infants remembered the associations the next day and sleep seems crucial for maintaining learning (Friedrich, Wilhelm, Born, \& Friederici, 2015; Friedrich, Wilhelm, Molle, Born, \& Friederici, 2017).

What do infants learn? A simple association or more than that? Naming an object helps children in many areas. Ten-month-old infants pay more attention to 
objects that have previously been named than to those that are silently presented or even pointed at (Baldwin \& Markman, 1989). It is not only attention that is amplified but also categorization of objects and their memorization. In a series of behavioral experiments in very young children, Waxman and collaborators studied the influence of language on the formation of conceptual categories. Different objects, or images, belonging to the same category are successively presented to children. The presentation is accompanied either by a sentence naming the object with a pseudoword "Look at the blicket", or by musical tones, or in silence, in different groups and ages. During the test, children were consistently much more able to distinguish between two new objects, one belonging to the familiar category and the other to a new category, in the naming condition (Waxman \& Markow, 1995; Balaban \& Waxman, 1997; Fulkerson \& Waxman, 2007; Ferry, Hespos, \& Waxman, 2010). These results suggest that naming invites children, as young as 3-month (Ferry, Hespos, \& Waxman, 2013) to form conceptual categories that they would not have considered without the use of words. This learning can be postponed and appear only after a sleep period: Only 6-8 month-old infants who nap generalize the name of an object to other exemplars of the same category (Friedrich et al., 2017; Friedrich et al., 2015).

Labelling an object with a word also allows infants to represent several objects. Before the age of 12 months, infants have difficulty maintaining the simultaneous representation of several objects. For example, when two objects appear alternately from the back of an opaque screen, infants do not seem to expect two objects when the screen is removed (Xu \& Carey, 1996). However, if each time the objects appear from behind the screen, they are named by two different words, infants are surprised when only one object is revealed. They are not surprised when the objects are designated by the same generic word "toy", or accompanied by two separate musical notes or sounds (Xu, 2002). The fact of naming each object specifically allows the individualization of the two objects.

Finally, labeling makes it possible to maintain more objects in working memory. In an experiment by Feigenson and Halberda (2008), four identical objects 
were hidden one by one in a box. The 14-month-old child must subsequently recover them, but two objects were surreptitiously removed by the experimenter. The time spent by the child searching for the two missing objects is then measured. In a first condition, the first two objects are named differently from the last two: "Look, a dax" then "Look, a blicket! " while in a second condition, each object is generically called to as "Look at this!". Children spend more time searching for missing objects when the experimenter has separated the 4 physically identical objects into 2 groups using 2 separate words than when he designates them with the same generic sentence. Young children can therefore use words to push the limits of their memory storage and thus memorize the 4 hidden objects, an ability that only appears much later in the absence of a name.

In these experiments, infants combine two interesting properties. They use speech as a source of valuable information about the world and use the label provided by speech to help them distinguish and memorize objects categories. What is the function of this label? Is it a powerful attention grabber because speech is a common and rewarding stimulus thanks to the social context it is embedded? Or does the label have a symbolic value, which enables to represent a category in a very compressed form that can be more easily handled in the internal working space?

\section{Are words symbols?}

In a symbolic system, there is an equivalence relationship between the set of symbols and the set of objects symbols stand for. Thus, unlike associative learning in which if $A$ is followed by $B$, it does not mean than $B$ is followed by $A$, in symbolic learning there is no direction between $A$ and $B$ because the object $A$ and the symbol $B$ point towards the same representation. Ekramnia and Dehaene-Lambertz (in preparation) have trained 4-month-old infants in a naming task of two categories of images (fribbles vs flying birds): a pseudo-word "kafon" was presented followed $1 \mathrm{~s}$ later by one of 180 images belonging to one category (e.g. birds) whereas "pauvou" was paired in the same way to the other category (e.g. fribbles). After 30 trials, $10 \%$ of incongruent trials with a mispairing were introduced to verify infant learning, but 
also $20 \%$ of reversed trials, in which the object was presented first followed by the name (in $10 \%$ of cases, the pairing was correct and in the other $10 \%$, incorrect). Incorrect pairs, whether in canonical or reversed trials, induced surprise responses in ERPs. Because infants have built an equivalence between the category and the name, reversed and canonical pairs are the two sides of the same coin, a process very different from associative learning which is directional. Kabdebon and Dehaene-lambertz (2019) went further and showed that infants are also able to name algebraic rules. In a series of experiments, 5-month-olds were trained to associate ever-changing trisyllabic nonce words characterized by the location of the repetition of a syllable (either immediate: AAB words or on the edges: ABA words) with an image (a fish or a lion). In the test, infants were surprised by incongruent pairings both in canonical and reversed trials.

This immediate generalization to reversed trials without further training is not observed in animals (Medam, Marzouki, Montant, \& Fagot, 2016), even chimpanzees (Kojima, 1984) and usually animals must learn separately both directions. It does not mean that symbols are not accessible to animals. For example, macaques can learn to represent quantities by abstract visual shapes, and they can even add these symbols and associate the result with the correct quantity (Livingstone et al., 2014; Srihasam, Mandeville, Morocz, Sullivan, \& Livingstone, 2012). However, the speed at which children learn these pairs and the spontaneous bi-directional mapping indicate another learning mechanism than simply slow associative learning, as previously assumed in infants (Nazzi \& Bertoncini, 2003). On the contrary, these recent experiments suggest that human infants assume an isomorphism between an internal symbolic space and the external world. Therefore, if the experimenter uses two words, the infant assumes that she must discover two kinds while one word implies that all the objects presented can be grouped together. Furthermore, infants' errors in Xu's (2002) and Feigenson and Halberda's (2008) studies may suggest that they are more attentive to symbolic representations than to sensory representations, probably because of its simpler manipulation and memorization, relative to the overwhelming richness of sensation. It might also 
reveal that the format of representations in the central workspace is symbolic, and that in explicit tasks, infants have access only to this format.

\section{Speech an information tool on the world}

If external information can be summarized by a symbol, what can be considered a symbol by infants? Waxman and colleagues showed, first, that it is not enough to couple an image with a sound for the sound to represent the image category; and second, that younger babies are more tolerant than older babies are. If speech and lemur vocalizations (but neither tones nor backward speech) are relevant for three-month-old infants, this is no longer the case for lemur vocalizations at 6 months (Ferry et al., 2013). At this older age, English-speaking babies are also not helped by a distant foreign language, such as Cantonese as opposed to a closer language such as German (Perszyk \& Waxman, 2019). It therefore seems that they are progressing not in their symbolic competence, but in their understanding of the communication medium accepted in their cultural environment.

Indeed, infants discover very early on that speech conveys information. In an eye-tracking experiment, Marno et al. (2015) presented 4-month-old infants with a video of an experimenter fixating them, then directing his gaze to the right (or left) where an object subsequently appears. Infants eyes moved more quickly towards the object when the experimenter was talking compared to a silent video or to a video accompanied by backward speech. Martin et al. (2012) presented a brief situation of interaction between two experimenters to 1-year-old children. During familiarization, a first person chooses one of the two objects presented, clearly indicating her preference for that object. In the next scene, the second experimenter faces the same two objects and interacts indifferently with each of them, without showing any preference. Finally, in the test phase, both people are present but the objects are out of reach of the first person. She turns to the second and coughs for a first group of children or says "koba" for a second group. The second experimenter then give her either her favorite object or the distractor. In the word, but not in the cough condition, children were surprised when the second experimenter did not give the first experimenter's favorite object and therefore look significantly longer. 1-year-old 
children therefore seem to expect that the information about the target object was conveyed between the two experimenters by a word, unlike cough noise. These results were subsequently replicated in 6-month-old infants (Vouloumanos, Martin, \& Onishi, 2014). Even with an attention grabbing and highly natural activity such as singing, 6 to 8-month-olds display less communicative behaviors (vocalization, visual contact, body movements and synchrony of these behaviors with maternal interactive behavior) in a face-to-face interaction than with a talking mother (Arias \& Pena, 2016). Infants therefore perceive the communication dimension of speech and its role as an information about the world.

If children have inferred that speech is a privileged channel of communication given their daily experience, they can also accept another medium of communication if social exchanges have depicted its use. For example, if they see two women exchanging with ostensive social signals but one speaking and the other "beeping", 6-month-old infants become able to use the "beeps" to identify a "dinosaur" category as opposed to "fish", unlike babies who have heard the same audio file but decorrelated from the social exchanges (Ferguson \& Waxman, 2016). This experiment illustrates the three key elements that support pedagogy in human infants: social cognition to figure out the communication medium, the ability to sort objects in categories, and a symbolic system to label any category. These three ingredients rely on different neural bases and co-develop during infancy. They allow infants to take advantage of other people' knowledge to identify the relevant information in the environment and thus boost their learning

If in the lab infants easily accept different types of labels (images, words, bips, etc..) to represent a category, in everyday life the situation is much more complex. Infants must find out how their cultural group communicates (for example oral or sign language), analyze this efficient but rich and complex communication system, and at the same time be attentive to the pertinent cues in their environment and correctly assign the correct label to the right object. The huge complexity of the task explains the apparent slowness of progress during the first years of life, and masks infants' early possibilities for symbolic representations. While early communication needs in 
infants, make language the first domain in which symbols are used, symbolic representations go beyond language in adults. It is interesting to note that mathematical knowledge and verbal knowledge are clearly separated in the adult brain (Amalric \& Dehaene, 2016) questioning on a general capacity for symbolic representations vs. an extension from an initial verbal domain to other domains.

\section{Conclusion}

To conclude, in this essay, we propose that teaching, an important activity for both parents and children from the first few months, requires summarizing information in a compressed abstract format for an effective sharing between individuals, and therefore implies symbolic representations. Although our hypothesis of symbolic representations in infants requires further experimental evidence, it parsimoniously explains several experimental results in the literature and is consistent with brain imaging observations that reveal a stronger continuity than previously thought in the functional cerebral architecture between infants and adults. The question of whether symbols are initially limited to the linguistic domain is an open question, but we may postulate that symbols extend beyond language and might represent the required representation of information in a conscious workspace.

Acknowledgment information: This work was supported by the Fondation de France, Fondation NRJ-Institut de France and the European Research Council (babylearn project).

\section{References}

Amalric, M., \& Dehaene, S. (2016). Origins of the brain networks for advanced mathematics in expert mathematicians. Proc Natl Acad Sci U S A, 113(18), 4909-4917.

Arias, D., \& Pena, M. (2016). Mother-Infant Face-to-Face Interaction: The Communicative Value of Infant-Directed Talking and Singing. Psychopathology, 49(4), 217-227.

Bahrami, B., Olsen, K., Latham, P. E., Roepstorff, A., Rees, G., \& Frith, C. D. (2010). Optimally interacting minds. Science, 329(5995), 1081-1085.

Balaban, M. T., \& Waxman, S. R. (1997). Do words facilitate object categorization in 9-month-old infants? Journal of Experimental Child Psychology, 64, 3-27.

Baldwin, D. A., \& Markman, E. M. (1989). Establishing word-object relations: a first step. Child Dev, 60(2), 381-398. 
Bergelson, E., \& Swingley, D. (2012). At 6-9 months, human infants know the meanings of many common nouns. Proc Natl Acad Sci U S A, 109(9), 3253-3258.

Chaplin, T. A., Yu, H. H., Soares, J. G., Gattass, R., \& Rosa, M. G. (2013). A conserved pattern of differential expansion of cortical areas in simian primates. J Neurosci, 33(38), 15120-15125.

Coqueugniot, H., Hublin, J. J., Veillon, F., Houet, F., \& Jacob, T. (2004). Early brain growth in Homo erectus and implications for cognitive ability. Nature, 431(7006), 299-302.

Csibra, G., \& Gergely, G. (2009). Natural pedagogy. Trends Cogn Sci, 13(4), 148-153.

d'Errico, F., \& Banks, W. E. (2015). The Archaeology of Teaching: A Conceptual Framework. Cambridge Archaeological Journal, 25(4), 859-866.

Dehaene-Lambertz, G., Dehaene, S., \& Hertz-Pannier, L. (2002). Functional neuroimaging of speech perception in infants. Science, 298(5600), 2013-2015.

Dehaene-Lambertz, G., Hertz-Pannier, L., Dubois, J., Meriaux, S., Roche, A., Sigman, M., \& Dehaene, S. (2006). Functional organization of perisylvian activation during presentation of sentences in preverbal infants. Proc Natl Acad Sci U S A, 103(38), 14240-14245.

Dehaene-Lambertz, G., Montavont, A., Jobert, A., Allirol, L., Dubois, J., Hertz-Pannier, L., \& Dehaene, S. (2010). Language or music, mother or Mozart? Structural and environmental influences on infants' language networks. Brain and Language, 114(2), 53-65.

Dehaene, S., \& Naccache, L. (2001). Towards a cognitive neuroscience of consciousness: basic evidence and a workspace framework. Cognition, 79(1-2), 1-37.

DeSilva, J., \& Lesnik, J. (2006). Chimpanzee neonatal brain size: Implications for brain growth in Homo erectus. J Hum Evol, 51(2), 207-212.

Ekramnia, M., \& Dehaene-Lambertz, G. (in preparation). Naming is more than creating an association: four-month-olds create equivalence relation between words and categories of objects

Emberson, L. L., Richards, J. E., \& Aslin, R. N. (2015). Top-down modulation in the infant brain: Learning-induced expectations rapidly affect the sensory cortex at 6 months. Proc Natl Acad Sci U S A, 112(31), 9585-9590.

Feigenson, L., \& Halberda, J. (2008). Conceptual knowledge increases infants' memory capacity. Proc Natl Acad Sci U S A, 105(29), 9926-9930.

Ferguson, B., \& Waxman, S. R. (2016). What the [beep]? Six-month-olds link novel communicative signals to meaning. Cognition, 146, 185-189.

Ferry, A. L., Hespos, S. J., \& Waxman, S. R. (2010). Categorization in 3- and 4-month-old infants: an advantage of words over tones. Child Dev, 81(2), 472-479.

Ferry, A. L., Hespos, S. J., \& Waxman, S. R. (2013). Nonhuman primate vocalizations support categorization in very young human infants. Proc Natl Acad Sci U S A, 110(38), 15231-15235.

Friedrich, M., \& Friederici, A. D. (2011). Word learning in 6-month-olds: fast encoding-weak retention. J Cogn Neurosci, 23(11), 3228-3240.

Friedrich, M., \& Friederici, A. D. (2017). The origins of word learning: Brain responses of 3-montholds indicate their rapid association of objects and words. Dev Sci, 20(2).

Friedrich, M., Wilhelm, I., Born, J., \& Friederici, A. D. (2015). Generalization of word meanings during infant sleep. Nat Commun, 6, 6004.

Friedrich, M., Wilhelm, I., Molle, M., Born, J., \& Friederici, A. D. (2017). The Sleeping Infant Brain Anticipates Development. Curr Biol, 27(15), 2374-2380 e2373.

Fulkerson, A. L., \& Waxman, S. R. (2007). Words (but not tones) facilitate object categorization: evidence from 6- and 12-month-olds. Cognition, 105(1), 218-228.

Gogate, L. J. (2010). Learning of syllable-object relations by preverbal infants: the role of temporal synchrony and syllable distinctiveness. J Exp Child Psychol, 105(3), 178-197. 
Gogate, L. J., Prince, C. G., \& Matatyaho, D. J. (2009). Two-month-old infants' sensitivity to changes in arbitrary syllable-object pairings: the role of temporal synchrony. J Exp Psychol Hum Percept Perform, 35(2), 508-519.

Gunz, P., Neubauer, S., Maureille, B., \& Hublin, J. J. (2010). Brain development after birth differs between Neanderthals and modern humans. Curr Biol, 20(21), R921-922.

Hermer-Vazquez, L., Spelke, E. S., \& Katsnelson, A. S. (1999). Sources of flexibility in human cognition: dual-task studies of space and language. Cognit Psychol, 39(1), 3-36.

Kabdebon, C., \& Dehaene-Lambertz, G. (2019). Symbolic labeling in 5-month-old human infants. Proc Natl Acad Sci U S A, 116(12), 5805-5810.

Kojima, T. (1984). Generalization Between Productive Use and Receptive Discrimination of Names in an Artificial Visual Language by a Chimpanzee. International Journal of Primatology, 5(2), 161-182.

Kouider, S., Long, B., Le Stanc, L., Charron, S., Fievet, A.-C., Barbosa, L. S., \& Gelskov, S. V. (2015). Neural dynamics of prediction and surprise in infants. Nature communications, 6.

Kouider, S., Stahlhut, C., Gelskov, S. V., Barbosa, L. S., Dutat, M., de Gardelle, V., . . DehaeneLambertz, G. (2013). A neural marker of perceptual consciousness in infants. Science, 340(6130), 376-380.

Livingstone, M. S., Pettine, W. W., Srihasam, K., Moore, B., Morocz, I. A., \& Lee, D. (2014). Symbol addition by monkeys provides evidence for normalized quantity coding. Proc Natl Acad Sci U S A, 111(18), 6822-6827.

Marno, H., Farroni, T., Vidal Dos Santos, Y., Ekramnia, M., Nespor, M., \& Mehler, J. (2015). Can you see what I am talking about? Human speech triggers referential expectation in four-monthold infants. Sci Rep, 5, 13594.

Medam, T., Marzouki, Y., Montant, M., \& Fagot, J. (2016). Categorization does not promote symmetry in Guinea baboons (Papio papio). Anim Cogn, 19(5), 987-998.

Mesulam, M. M. (1998). From sensation to cognition. Brain, 121, 1013-1052.

Nazzi, T., \& Bertoncini, J. (2003). Before and after the vocabulary spurt: two modes of word acquisition? Developmental Science, 6(2), 136-142.

Neubauer, S., Gunz, P., \& Hublin, J. J. (2010). Endocranial shape changes during growth in chimpanzees and humans: a morphometric analysis of unique and shared aspects. $J$ Hum Evol, 59(5), 555-566.

Neubauer, S., Hublin, J. J., \& Gunz, P. (2018). The evolution of modern human brain shape. Sci Adv, 4(1), eaao5961.

Neubert, F. X., Mars, R. B., Thomas, A. G., Sallet, J., \& Rushworth, M. F. (2014). Comparison of human ventral frontal cortex areas for cognitive control and language with areas in monkey frontal cortex. Neuron, 81(3), 700-713.

Perszyk, D. R., \& Waxman, S. R. (2019). Infants' advances in speech perception shape their earliest links between language and cognition. Sci Rep, 9(1), 3293.

Price, D. J., Kennedy, H., Dehay, C., Zhou, L., Mercier, M., Jossin, Y., . . Molnar, Z. (2006). The development of cortical connections. Eur J Neurosci, 23(4), 910-920.

Sakai, T., Hirata, S., Fuwa, K., Sugama, K., Kusunoki, K., Makishima, H., .. Takeshita, H. (2012). Fetal brain development in chimpanzees versus humans. Curr Biol, 22(18), R791-792.

Sakai, T., Mikami, A., Tomonaga, M., Matsui, M., Suzuki, J., Hamada, Y., . . Matsuzawa, T. (2011). Differential prefrontal white matter development in chimpanzees and humans. Curr Biol, 21(16), 1397-1402.

Srihasam, K., Mandeville, J. B., Morocz, I. A., Sullivan, K. J., \& Livingstone, M. S. (2012). Behavioral and anatomical consequences of early versus late symbol training in macaques. Neuron, 73(3), 608-619. 
Tincoff, R., \& Jusczyk, P. W. (1999). Some beginnings of word comprehension in 6-month-olds. Psychological Science, 10(2), 172-175.

Tincoff, R., \& Jusczyk, P. W. (2012). Six-Month-Olds comprehend words that refer to parts of the body. Infancy, 17(4), 432-444.

Vouloumanos, A., Martin, A., \& Onishi, K. H. (2014). Do 6-month-olds understand that speech can communicate? Dev Sci, 17(6), 872-879.

Wang, L., Uhrig, L., Jarraya, B., \& Dehaene, S. (2015). Representation of numerical and sequential patterns in macaque and human brains. Curr Biol, 25(15), 1966-1974.

Waxman, S. R., \& Markow, D. B. (1995). Words as invitations to form categories: Evidence from 12to 13-month-old infants. Cognitive Psychology, 29, 257-302.

$\mathrm{Xu}, \mathrm{F}$. (2002). The role of language in acquiring object kind concepts in infancy. Cognition, 85(3), 223250.

Xu, F., \& Carey, S. (1996). Infants' metaphysics: The case of numerical identity. Cognitive Psychology, 30(2), 111-153. 